\title{
Extramammary Paget's Disease Covered the Left Nipple and Areola
}

\author{
Ikuko Abe $^{1 *}$, Kazuyoshi Sugiyama ${ }^{2}$, Masaru Suda $^{2}$, Fumie Igari ${ }^{2}, K_{\text {Koji Senuma }}{ }^{1}$, Atsushi Arakawa ${ }^{3}$, \\ Mitsue Saito ${ }^{1}$, Fujio Kasumi ${ }^{1}$
}

${ }^{1}$ Department of Breast \& Endocrine Surgery, School of Medicine, Juntendo University, Tokyo, Japan; ${ }^{2}$ Department of Surgery, Juntendo University School of Medicine Urayasu Hospital, Chiba, Japan; ${ }^{3}$ Department of Human Pathology, Juntendo University School of Medicine, Tokyo, Japan.

Email: *iabe@juntendo.ac.jp

Received October $26^{\text {th }}, 2011$; revised November $24^{\text {th }}, 2011$; accepted December $26^{\text {th }}, 2011$

\begin{abstract}
We present a case of a 71-year-old woman suffering from mammary Paget's disease and having a 10-years history of an irregular, widespread erosion accompanied by itching and burning on the skin of her left chest, extending to the breast. The erosion had steadily enlarged and had become increasingly tender. The nipple and areola of the left breast disappeared and could not be recognized. No abnormality of the right nipple, areola, and covering skin and no supernumerary nipple were seen. Mammography and ultrasonography could not be performed because of severe pain and erosive wetness. Histopathology of a surgical biopsy specimen showed epidermal infiltration by large, round, clear atypical cells scattered individually or in small clusters and distributed horizontally throughout the epidermis. The cytoplasm of these large cells was pale and vacuolated and was equivalent to that in nipple cells in Paget's disease, and a diagnosis of mammary Paget's disease was made. We performed total mastectomy of the left breast with wide excision of the Paget lesion of the left chest and axillary lymph node sampling. Histological examination of the specimen showed typical distribution of Paget's cells; however no ductal carcinoma in situ was found in the mammary ducts and invasive growth was not recognized beyond the basal membrane of the lesion. From this evidences, we established a diagnosis of large, irregulaly shaped unusual mammary Paget's disease, not of breast cancer origin, covering the left breast, areola, and nipple.
\end{abstract}

Keywords: Mammary Paget’s Disease; Extramammary Paget’s Disease; Nipple; Areola

\section{Introduction}

Mammary Paget's disease (MPD) is common and well known condition breast cancer affecting the nipple and areola.

On the other hand extramammary Paget's disease (EMPD) has been described to commonly occur in the skin of the genital, perianal, perineal, and axillary regions $[1,2]$. However unusual cases arising on the face [3], cheek [4], upper abdomen [5,6], lower anterior chest [7,8], lateral chest [8], and scalp [9] have been reported. We describe a type of unusual MPD affecting the lateral half of the left breast and adjacent chest skin. As the lesion was irregular in shape large size without underlying carcinoma, and the left nipple and areola were covered and had disappeared. It was difficult to distinguish EMPD from MPD. To our knowledge, only a few reports similar to ours have been reported previously. We describe this case and discuss its unique clinical and, pathological

${ }^{*}$ Corresponding author. features.

\section{Case Report}

A 71-year-old woman presented with a $20 \times 10 \mathrm{~cm}$ sharp widespread erosion on the lateral side of her left breast and adjacent chest skin (Figure 1). She had noted an erythematous erosion in her left side breast 10 years ago, which steadily enlarged and became increasingly tender. She complained of itching and burning. Physical examination revealed an erythema, erosion, and mixed pigmentation as well as hypopigmentation. The skin change was sharply demarcated, however, it had irregular margins. The nipple and areola of the left breast were covered by the lesion and had disappeared. No abnormality in the right nipple or its covering skin was observed. No axillary lymphadenopathy was noted. Mammography and ultrasonography could not be performed because of severe pain and erosive wetness. Chest and abdominal CT scans showed no abnormal findings. The clinical 


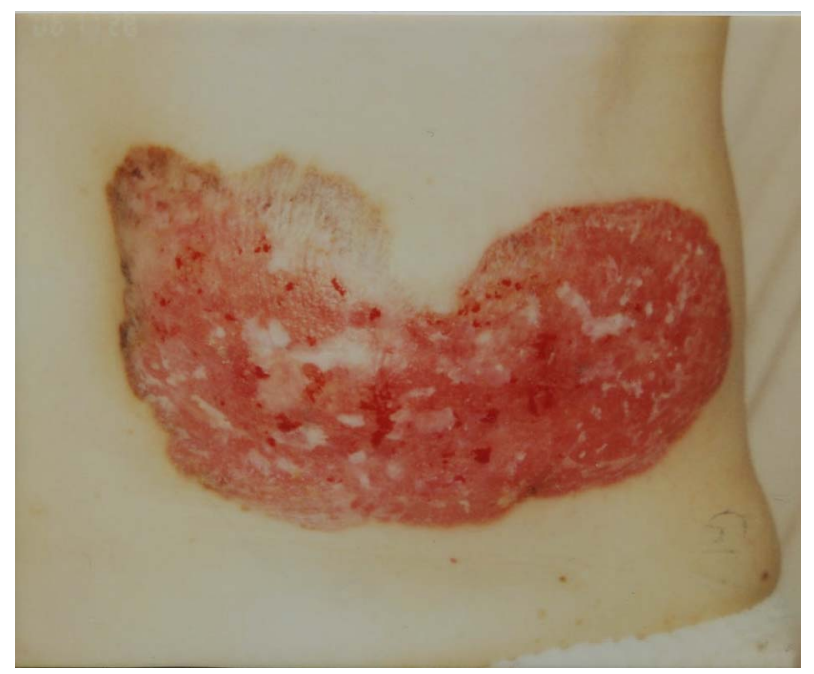

Figure 1. Sharply demarcated $20 \times 10 \mathrm{~cm}$ erosion erythematous erosion in the left breast with pigmentation and hypopigmentation.

findings were suggestive of MPD but the location, size, and irregular spread, which did not make a centrifugal circle around the nipple, were somewhat abnormal compared with usual MPD appearance. Histopathology of a surgical biopsy specimen of the skin lesion however, showed typical Paget cells forming irregular nests and scattering individually in the epidermis (Figure 2(a)). We confirmed as a MPD of the left breast, and performed a total mastectomy with wide skin resection and axillary lymph node sampling without a skin graft. The histological findings were similar to these of the biopsy specimens. Paget cells were in the epidermis and chronic inflammatory reaction indications, mainly lymphocytes, and aggregation of abundant telangiectasia were seen in the upper dermis (Figure 2(b)). The mammary gland showed very atrophic and mildly sclerotic stroma, there is no focus of invasive ductal carcinoma or ductal carcinoma in situ (DCIS) lesions and minute calcifications were absent (Figure 2(c)). Supernumerary nipple was absent. The dissected lymph nodes were free of tumors. Immunohistochemical findings revealed that Paget cells were positive for PAS, CK7, Ki67, PgR and Her2, but negative for ER, CK20, gross cystic disease fluid protein (GCDFP)-15, polyclonal carcinoembryonic antigen (CEA), and staining with alcian blue. Two-years after operation, the patient showed no evidence of recurrence.

\section{Discussion}

EMPD is a rare condition and has a predilection for areas with high apocrine gland density, such as the vulva, perianal area, scrotum, penis and axilla [1]. Usually most cases in which a Paget lesion is found. EMPD is a secondary event caused by adenocarcinoma extension of adjacent other organs [1]. Typical isolated skin EMPD

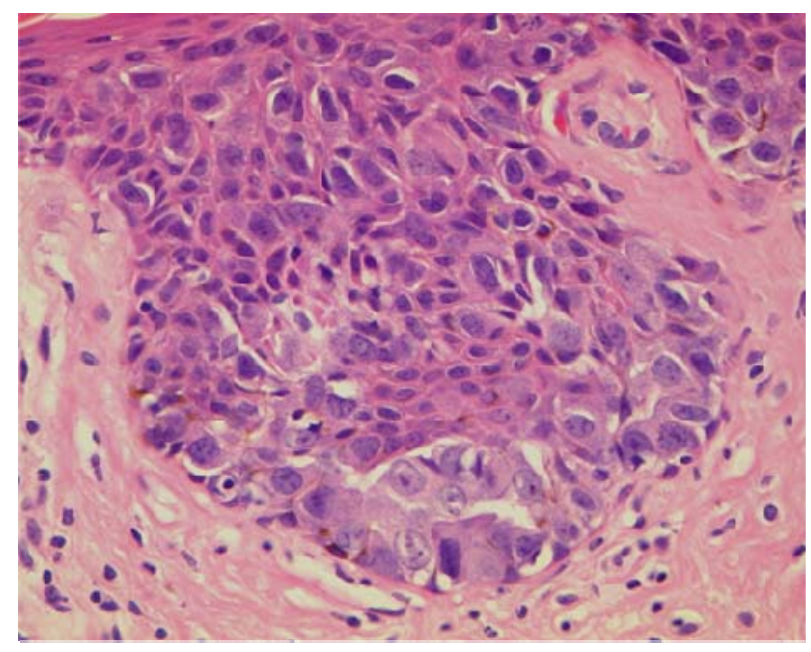

(a)

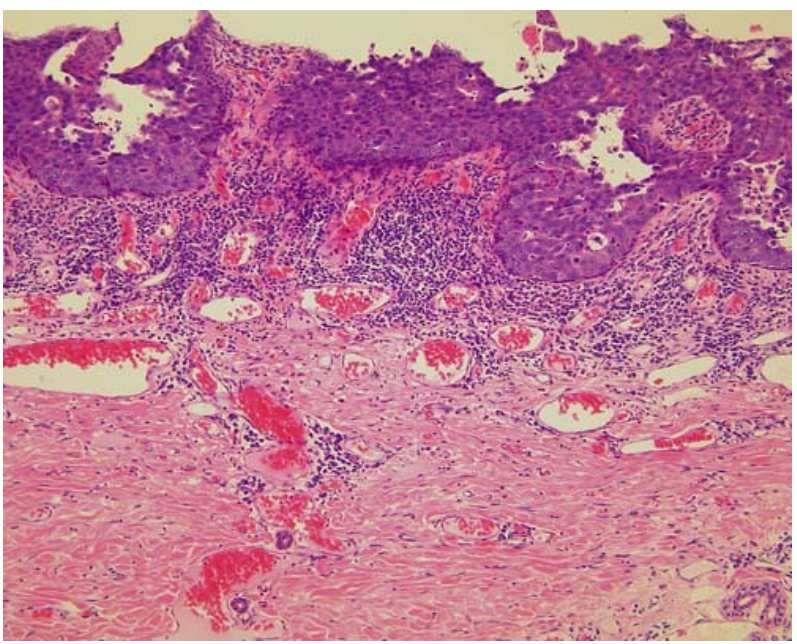

(b)

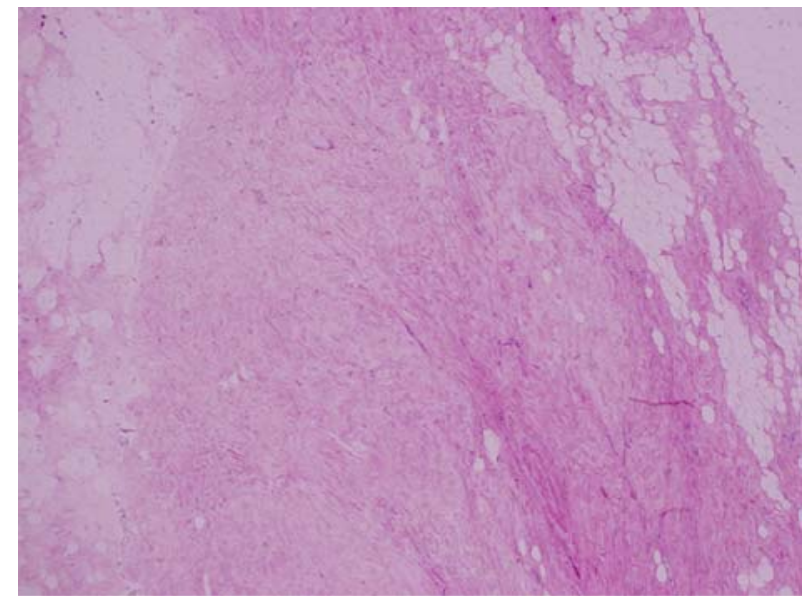

(c)

Figure 2. (a) Histopathologic features of extramammary Paget's disease. Pagetoid tumor cells are distributed within the epidermis with sparse meranin; (b) No dermis invasion was observed; (c) There is no focus of intraductal carcinoma or DCIS. 
presents moist and superficially eroded or erythematous scaly patches; hypopigmented macules, ulceration, and crusting may also be present [2]. The coexistence of pigmentary disorders (pigmentation and/or depigmentation) with these features has also been observed by clinicians [10]. The most common symptom is pruritus, and the duration of symptoms prior to diagnosis varies from a few months to more than 10 years [11]. EMPD usually presents as a slowly expanding erythematous change, with sharp demarcation and an erythematous scaly surface. Occurrence in another sites is rare. Saida et al. [7] proposed that such a condition should be called ectopic EMPD. They found that the lesion may originate in the ectopic apocrine sweat glands or develop from the pluripotent epidermis matrix cells [12]. Precise mechanism of EMPD incidence remains unknown because its frequency is lower than that of MPD. Moreover in the literature to date, there are 5 reported cases of EMPD extending to the breast and nipple of the anterior chest [7,13-16]. EMPD affecting the ipsilateral breast and nipple is a very rare condition, whereas MPD of the normal breast originates from DCIS of mammary ducts and spreads on the nipple surface and extends slowly to the areola and surrounding skin, usually in a centrifugal circle [17]. MPD is associated with an underlying DCIS of the breast [18]. In the present case, a no supernumerary nipple was found and the adjacent skin was involved [19]. Since the rate of spread the lesion located at nipple to the surrounding areola changes is slow, usually several $\mathrm{mm}$ in diameter par year, patients often wait a year or more and in rare case over 10 years, before seeking advice from a doctor [1]. In the literature to date, there has been no case of MPD where the nipple has disappeared [18,19]. In our case, clinicopathologically there is no nipple and areola and, there is no invasive carcinoma or DCIS in the mammary gland. Horii et al. reported spontaneous healing which the intraductal component of breast cancer disappears and is replaced by fibrous tissue. The proportion of cases in which healing was recognized Paget's disease was $50 \%$ [20]. We could not think of taking independent healing phenomenon.

Thirteen patients with MPD have undergone surgical treatment in our hospital during a 6 year period from 2003 to 2008; representing $0.9 \%$ of 1505 cases of breast cancers. All cases were in women, and MPD in bilateral breasts was absent. In all 13 cases, the nipple and areola had almost disappeared and were recognizable in images. However according to standard textbooks of dermatology [21], EMPD are usually long large standing and, the affecting nipple may be falling off. On the other hand extensive MPD has rarely been described in case reports [22]. In 2007 Kanwar et al. [23] reported a woman presenting a 14-year history of an eczematous lesion. The lesion started from the left nipple and extended over the breast left axilla to the and across the nipple of the right breast. However, in MPD, the nipple does not usually fall off. However, it is also known that in some cases of MPD it is not possible to find breast cancer in the ipsilateral mammary gland.

Immunohistochemical techniques can also be used to identify Paget's cells. Generally, these cells are stained by CEA [24], low-molecular-weight cytokeratins [25], and epithelial membrane antigen [26]. Paget cells are also stained by GCDFP-15, which is believed to be apocrine cell specific; however, it may localize to eccrine cells. Typically, in MPD, Paget cells are CK7-positive, whereas in EMPD extending to the nipple, cells may be CK20-positive [27]. Strong Her2/neu oncoprotein immunoreactivity has been detected in $79 \%$ to $100 \%$ of MPD samples studied; however, it has been detected in only $0 \%$ to $40 \%$ of EMPD cases [19]. The proliferation of the Ki-67 index in Paget cells in MPD cases is relatively similar to that of underlying intraductal carcinoma [28]. In our patient, GCDFP-15, CEA, and alcian blue were negative, thus it did not support differential diagnosis of MPD and EMPD.

To the best of our knowledge, there has been no previous report similar to ours. From our experience, Paget's disease on the breast is usually a MPD; however, we must also we must remember that a rare case of breast EMPD is also possible. In rare cases, EMPD may affect the nipple or the areola from the outer, anterior, or lateral side of the chest. Therefore, in these cases, mastectomy may not be made and prevent excessive treatment. Until now, MPD has been treated by breast surgeons, while EMPD has been treated by dermatologists. We wish to emphasize that building a closer connection between breast surgeons and dermatologists is a necessity.

\section{REFERENCES}

[1] R. M. Mackie, "Paget's Disease of the Nipple. Extramammary Paget's Disease,” In: R. H. Champion, J. L. Burton, D. A. Burns and S. M. Breathnach, Eds., Textbook of Dermatology (Rook, Wilkinson, Ebling), 6th Edition, Blackwell Scientific Publications, Oxford, 2005. pp. 1677-1679.

[2] S. M. Connolly, "Mammary and Extramammary Paget's Disease,” In: I. M. Freedberg, A. Z. Eisen, K. Wolff, K. F. Austen, Eds., Dermatology in General Medicine, 6th Edition, McGraw-Hill, New York, 2003. pp. 813-817.

[3] M. A. Cohen, A. Hanly, E. Poulos and G. D. Goldstein, "Extramammary Paget's Disease Presenting on the Face," Dermatologic Surgery, Vol. 30, No. 10, 2004, pp. 13611363. doi:10.1111/j.1524-4725.2004.30412.x

[4] S. Chilukuri, R. Page, J. A. Reed, J. Friedman and I. Orengo, "Ectopic Extramammary Paget's Disease Arising on the Cheek,” Dermatologic Surgery, Vol. 28, No. 5, 2002, pp. 430-433. doi:10.1046/j.1524-4725.2002.01199.x

[5] Y. Onishi and K. Ohara, "Ectopic Extramammary Paget’s 
Disease Affecting the Upper Abdomen,” British Journal of Dermatology, Vol. 134, No. 5, 1996, pp. 958-961. doi:10.1111/j.1365-2133.1996.tb06336.x

[6] Y. Nagai, H. Ishibuchi, M. Takahashi, A. Shimizu, Y. Matushima, A. Tamura, et al., "Extramammary Paget’s Disease with Bowenoid Histologic Features Accompanied by an Ectopic Lesion on the Upper Abdomen," Journal of Dermatology, Vol. 32, No. 8, 2005, pp. 670-673.

[7] T. Saida and M. Iwata, “'Ectopic' Extramammary Paget's Disease Affecting the Lower Anterior Aspect of the Chest," Journal of the American Academy of Dermatology, Vol. 17, No. 5, 1987, pp. 910-913. doi:10.1016/S0190-9622(87)70279-5

[8] M. H. Yeh and S. L. Hu, "Treatment of Double Ectopic Extramammary Paget's Disease of Bilateral Chest with Imiquimod 5\% Cream," Journal of the European Academy of Dermatology and Venereology, Vol. 21, No. 7, 2007, pp. 997-999. doi:10.1111/j.1468-3083.2006.02070.x

[9] O. H. Iwenofu, F. H. Samie, J. Ralston, R. T. Cheney and N. C. Zeitouni, "Extramammary Paget's Disease Presenting as Alopecia Neoplastica,” Journal of Cutaneous Pathology, Vol. 35, No. , 2008, pp. 761-764. doi:10.1111/j.1600-0560.2007.00897.x

[10] H. Kakinuma, U. Iwasawa, N. Kurakata and H. Suzuki, "A Case of Extramammary Paget's Disease with Depigmented Macules as the Sole Manifestation," British Journal of Dermatology, Vol. 130, No. 1, 1994, pp. 102-105. doi:10.1111/j.1365-2133.1994.tb06892.x

[11] R. E. Jones Jr., C. Austin and A. B. Ackerman, "Extramammary Paget's Disease. A Critical Reexamination," The American Journal of Dermatopathology, Vol. 1, No. 2, 1997, pp. 101-132. doi:10.1097/00000372-197900120-00002

[12] T. S Saunders, "Extramammary Paget's Disease and the Milk Line” Journal of the American Academy of Dermatology, Vol. 19, No. 1, 1988, p. 139. doi:10.1016/S0190-9622(88)80231-7

[13] T. Takeuchi, M. Komatsuzaki, Y. Minesaki, K. Yokoi, R. Kamide, M. Niimura, et al., "Paget's Disease Arising near a Male Areola without an Underlying Carcinoma,” Journal of Dermatology, Vol. 26, No. 4, 1999, pp. 248-252.

[14] S. Ito, K. Arai, M. Saitoh, Y. Kato, T. Ohi and M. Koga, "A Case of Extramammary Paget's Disease Affecting the Left Lateral Chest,” Nishinihonhifuka, Vol. 65, No. 4, 2003, pp. 343-346. doi:10.2336/nishinihonhifu.65.343

[15] H. Miyaguni, S. Sakaki, M. Kurokawa, K. Ogata and S. Inoue, "A Case of Mammary Paget's Disease without an Underlying Carcinoma,” Hifukanorinsho, Vol. 42, No. 8, 2000, pp. 1187-1190.

[16] K. E. Taylor, H. M. Ormsby, J. M. Havercroft and A. Rodger, “An Unusually Extensive Case of Paget's Disease of the Nipple,” The Breast, Vol. 10, No. 5, 2001, pp.
442-446. doi:10.1054/brst.2000.0268

[17] F. Kasumi, “Kasumi’s Color Atlas of Breast Cancer,” Igakushoin, 2009, pp. 259-272.

[18] G. H. Sakorafas, K. Blanchard, M. G. Sarr and D. R. Farley, "Paget's Disease of the Breast," Cancer Treatment Reviews, Vol. 27, No. 1, 2001, pp. 9-18. doi:10.1053/ctrv.2000.0203

[19] P. P. Rosen, “Paget's Disease of the Nipple,” In: P. P. Rosen, Ed., Rosen's Breast Pathology, Lippincott Williams \& Wilkins, Philadelphia, 2001, pp. 565-759.

[20] R. Horii, F. Akiyama, F. Kasumi, M. Koike and G. Sakamoto, "Spontaneous 'Healing' of Breast Cancer," Breast Cancer, Vol. 12, No. 2, 2005, pp. 140-144. doi:10.2325/jbcs.12.140

[21] Y. Kawabata, "Mammary Paget's Disease and Extramammary Paget's Disease,” In: K. Tamaki, H. Iizuka, H. Shimizu, et al., Eds., Handbook of Dermatology, Vol. 12. Nakayama Shoten, Tokyo, 2002, pp. 216-229. doi:10.1016/j.breast.2003.11.005

[22] G. Nicoletti, S. Scevola, R. Ruggiero, A. M. Coghi and G. S. Toussoun, "Gigantic Paget Disease of the Breast," The Breast, Vol. 13, No. 5, 2004, pp. 425-427.

[23] A. J. Kanwar, D. De, K. Vaiphei, A. Bhatia, R. K. Sharma and G. Singh, "Extensive Mammary Paget's Disease," Clinical and Experimental Dermatology, Vol. 32, No. 3, 2007, pp. 326-327. doi:10.1111/j.1365-2230.2006.02326.x

[24] O. Mori, H. Hachisuka and Y. Sasai, "Immunohistochemical Demonstration of Epithelial Membrane Antigen (EMA), Carcinoembryonic Antigen (CEA), and Keratin on Mammary and Extramammary Paget's Disease," Acta Histochemitry, Vol. 85, No. 1, 1989, pp. 93-100.

[25] T. Tazawa, M. Ito, H. Fujiwara, N. Shimizu and Y. Sato, "Immunologic Characteristics of Keratins in Extramammary Paget's Disease," Archives of Dermatology, Vol. 124, No. 7, 1988, pp. 1063-1068. doi:10.1001/archderm.1988.01670070051018

[26] M. A. Nowak, P. Guerriere-Kovach, A. Pathan, T. E. Campbell and L. M. Deppisch, "Perianal Paget's Disease: Distinguishing Primary and Secondary Lesions Using Immunohistochemical Studies Including Gross Cystic Disease fluid Protein-15 and Cytokeratin 20 Expression,” Archives of Pathology Laboratory Medicine, Vol. 122, No. 12, 2000, pp. 1077-1081.

[27] N. Kirkham, "Paget's Disease. Extramammary Paget's Disease,” In: D. Elder, Ed., Lever's Histopathology of the Skin, 9th Edition, New York Lippincott-Raven, Philadelphia, 2005, pp. 852-855.

[28] J. C. Noel, I. Fayt and F. Buxant, "Proliferating Activity in Paget Disease of the Nipple," Pathology and Oncology Research, Vol. 16, No. 1, 2010, pp. 7-10. doi:10.1007/s12253-009-9179-4 